\title{
Oscilador forçado por um pente de Dirac: uma aplicação da transformada de Fourier-Mellin
}

\author{
Forced oscillator by a Dirac comb: an application of the Fourier-Mellin transform \\ Rafael de Sousa Dutra ${ }^{*}\left[\right.$, Laercio Costa Ribeiro ${ }^{2}$, Claudio Maia Porto $^{3}$ \\ ${ }^{1}$ Instituto Federal de Educação, Ciência e Tecnologia, Paracambi, RJ, Brasil \\ ${ }^{2}$ Centro Federal de Educação Tecnológica Celso Suckow da Fonseca, Nova Iguaçu, RJ, Brasil \\ ${ }^{3}$ Universidade Federal Rural do Rio de Janeiro, Departamento de Física, Seropédica, RJ, Brasil
}

Recebido em 12 de Maio, 2018. Revisado em 24 de Junho, 2018. Aceito em 25 de Junho, 2018.

\begin{abstract}
Neste trabalho exploramos a técnica da transformada de Laplace inversa, conhecida como transformada de Fourier-Mellin, para solucionar, de forma direta e rigorosa, o problema de um sistema que oscila sob a ação de uma força externa periódica. Também propomos um modelo em que essa força externa é descrita por uma sucessão de deltas de Dirac, uma estrutura conhecida na literatura como pente de Dirac. Esse modelo é adequado para descrever o problema clássico de uma criança sendo impulsionada em um balanço, usualmente descrito em termos de uma força externa senoidal. Indicamos nosso modelo de oscilador forçado como mais realista na descrição desse tipo de problema por considerar a atuação da força externa apenas no intervalo que corresponde ao tempo de contato entre a criança e o agente externo que realiza a força, intervalo esse que tende a zero. O principal resultado deste trabalho foi obtido no regime de ressonância, no qual a potência média transferida ao sistema apresentou uma série de picos, associados aos múltiplos inteiros da frequência natural de oscilação, diferente do que ocorre no caso ordinário, em que a força externa é descrita por uma função trigonométrica.
\end{abstract}

Palavras-chave: Mecânica clássica; Transformada de Fourier-Mellin; Oscilações forçadas.

In this work we explore the technique of the inverse of the Laplace transform, which is known as the FourierMellin transform, to solve, in a direct and rigorous way, the problem of a system that oscillates under the action of a periodic external force. We also present a model in which the external force is described in terms of a sequence of Dirac deltas. This model is pertinent to describe the classical problem of a child boosted in a park swing, usually described in terms of a sinusoidal function. We indicate our forced oscillator model as more realistic to the description of this kind of problem as it considers the action of the external force only during the very small time interval of contact between the child and the agent that applies the force. The principal result of this paper were obtained in the resonance regime, in which the average power transferred to the system presented a series of peaks, corresponding to the entire multiples of the natural frequency of oscillation, in contrast to what is obtained in the ordinary case in which the external force is described by a trigonometric function.

Keywords: Classical mechanics; Fourier-Mellin transform; Forced oscillations.

\section{Introdução}

A partir das leis fundamentais da dinâmica estabelecidas por Isaac Newton em fins do século XVII é possível obter de forma determinística as grandezas físicas, tais como posição, velocidade e aceleração, associadas ao movimento de corpos que possuam dimensões relativamente grandes, comparadas às dimensões da escala atômica e molecular, e que se locomovam a velocidades relativamente baixas, comparadas à da luz. De forma específica, a aplicação da segunda lei de Newton na descrição do movimento de um corpo de massa $m$ submetido à ação de forças externas resulta em uma equação diferencial que, dependendo da complexidade das forças envolvidas, e sendo conhecidas as condições iniciais obedecidas pelo sistema, pode ser

*Endereço de correspondência: rafael.dutra@ifrj.edu.br resolvida de modo a fornecer uma expressão analítica para a dependência temporal das grandezas em questão.

Entre os diferentes tipos de movimento que podem ser estudados destacamos o movimento oscilatório, no qual um sistema é posto a oscilar em torno de sua posição de equilíbrio estável sob a ação de uma força restauradora, cuja ação se dirige a trazer o sistema novamente ao ponto de equilíbrio. A importância especial dada a esse problema se deve ao fato de que, independente da força conservativa que atue sobre um determinado corpo, por mais complexa que seja sua forma matemática, para pequenos deslocamentos em relação à posição de equilíbrio, podemos aproximá-la por uma força restauradora proporcional a esse deslocamento, fazendo dessa situação, aparentemente restritiva, um paradigma geral. Frequentemente esse movimento oscilatório é afetado pela ação 
de forças dissipativas significativas e/ou pela ação de uma força externa aplicada.

Entre as modalidades de movimentos oscilatórios, o movimento oscilatório forçado $\sqrt{1 / 3}]$ é certamente o mais interessante pela riqueza da física incorporada ao sistema através da força externa aplicada, que, em geral, depende do tempo. Em sua versão mais completa, na qual são incluídos os efeitos dissipativos, esse movimento é descrito por uma equação diferencial não homogênea, cujo termo independente é associado à força externa aplicada. Tradicionalmente propõe-se um modelo em que a parte dissipativa dessa equação é incluída através de uma dependência linear com a velocidade, enquanto a força externa depende do tempo através de uma função senoidal ${ }^{1}$ A solução dessa equação é geralmente obtida pela composição de uma solução referente à parte homogênea com outra referente à solução particular estacionária da equação inomogênea, solução esta que descreve a dinâmica do sistema após um tempo suficientemente grande, contado a partir do início do movimento.

Uma forma mais direta de se resolver uma equação diferencial desse tipo é utilizando a metodologia das transformadas de Laplace, que consiste em aplicar o operador Laplaciano $\mathcal{L}$ em cada um dos termos da equação diferencial, para em seguida obter uma solução no espaço complexo, e, por fim, retornar com a solução para o espaço real através da transformada de Laplace inversa, no plano complexo, conhecida como transformada de Fourier-Mellin [4].

Neste artigo exploramos o método das transformadas de Laplace para obter a solução da equação diferencial associada a um movimento oscilatório menos tradicional, no qual a força externa é descrita por uma série de "funções"delta de Dirac 5. 6], representando, por exemplo, o sistema composto por uma criança sendo periodicamente impulsionada em um balanço por uma força externa que atua em intervalos de tempo extremamente pequenos.

Na segunda seção deste trabalho apresentamos a metodologia das transformadas de Laplace inversas aplicadas na solução de equações diferenciais inomogêneas associadas a osciladores forçados. Na terceira seção aplicamos essa metodologia ao problema específico proposto e na quarta e última seção apresentamos as considerações finais, com as conclusões sobre os resultados obtidos.

\section{O Método de Laplace}

Consideremos o movimento de translação de um corpo de massa $m$ preso a uma mola de constante elástica $k$ que, para pequenos deslocamentos, exerce uma força restauradora que obedece à lei de Hooke, $F_{\text {rest }}=-k x$. Imaginemos todo esse sistema imerso em um meio viscoso, cuja

\footnotetext{
1 Há uma justificativa didática para esse tratamento: as funções senoidais constituem uma base em termos da qual podemos escrever os mais variados tipos de funções, de modo que, se soubermos resolver o problema na situação em que o termo independente é senoidal, os problemas mais gerais serão resolvidos em termos de superposições desses casos.
}

força de amortecimento modelamos como sendo proporcional à velocidade, $F_{\text {amort }}=-b v$, onde a constante de proporcionalidade $b$ depende da aerodinâmica do corpo e o sinal negativo indica que o sentido de atuação da força se opõe ao da velocidade. Esse sistema, posto a oscilar por meio de uma força externa dependente do tempo $F(t)$, é conhecido como oscilador amortecido forçado.

Considerando um movimento retilíneo ao longo da direção X, a aplicação da segunda de lei de Newton fornece a equação de movimento para esse sistema: $-k x-$ $b v+F(t)=m a$. Expressando a velocidade e a aceleração em termos das derivadas temporais da posição, $v(t)=$ $d x / d t=\dot{x}$ e $a(t)=d^{2} x / d t^{2}=\ddot{x}$, obtemos 7

$$
\ddot{x}+\beta \dot{x}+\omega_{o}^{2} x=F(t) / m,
$$

sendo $\beta$, igual a $b / m$, o coeficiente de amortecimento, e $\omega_{o}$, igual a $\sqrt{k / m}$, a frequência natural de oscilação do sistema. É importante mencionar que uma equação análoga pode ser obtida no estudo de circuitos elétricos oscilantes sob a ação de picos periódicos de tensão e que a metodologia aqui descrita pode ser aplicada a tais sistemas.

Definimos o operador linear Transformada de Laplace de uma função $f(t)$, representado por $\mathcal{L}\{\}$, como

$$
\mathcal{L}\{f(t)\}=G(s)=\int_{0}^{\infty} f(t) e^{-s t} d t, t>0,
$$

Aplicando-o a ambos os lados da equação (1), temos

$$
\mathcal{L}\{\ddot{x}\}+\beta \mathcal{L}\{\dot{x}\}+\omega_{o}^{2} \mathcal{L}\{x\}=\mathcal{L}\{F(t)\} / m .
$$

Realizando, em seguida, algumas integrações por partes, temos que $\mathcal{L}\{x\}=X(s), \mathcal{L}\{\dot{x}\}=s X(s)-x_{0}$ e $\mathcal{L}\{\ddot{x}\}=s^{2} X(s)-s x_{0}-v_{0} \quad 8$. Obtemos assim a chamada função de transferência do sistema, dada por

$$
X(s)=\frac{x_{0} s+\left(v_{0}+\beta x_{0}\right)}{s^{2}+\beta s+\omega_{0}^{2}}+\frac{F(s)}{m\left(s^{2}+\beta s+\omega_{0}^{2}\right)},
$$

onde $x_{0}$ e $v_{0}$ representam, respectivamente, a posição e a velocidade inicial do oscilador, com $F(s)$ sendo a transformada de Laplace da força externa, $\mathcal{L}\{F(t)\}=$ $F(s)$.

Para obter a solução final, é preciso efetuar a transformada de Laplace inversa da função de transferência $X(s)$ dada pela equação (4). Costuma-se obter essa inversão decompondo a transformada de Laplace em funções cujas transformadas são conhecidas e recorrendo ao auxílio de uma tabela. Todavia, como podemos notar a partir da referida equação, nem sempre será uma tarefa fácil a decomposição de $X(s)$ em funções com transformadas conhecidas, uma vez que a função $F(s)$ pode ser a mais geral possível. 
Para solucionar o problema de maneira rigorosa, utilizamos a inversa da expressão (2), conhecida como transformada de Fourier-Mellin, dada pela integral de Bromwich no plano complexo $[9]$ :

$$
\mathcal{L}^{-1}\{G(s)\}=f(t)=\frac{1}{2 \pi i} \lim _{w \rightarrow \infty} \int_{\gamma-i w}^{\gamma+i w} G(s) e^{s t} d s,
$$

onde a integração é realizada numa linha vertical, $\Re(s)=$ $\gamma$, no plano complexo, de tal modo que $\gamma$ deve ser maior do que a parte real de todas as singularidades de $G(s)$, uma vez que, para aplicarmos o teorema de Cauchy, devemos utilizar um percurso fechado à esquerda dessa linha vertical, envolvendo essas singularidades.

Substituindo a expressão de $X(s)$ dada pela equação (4) no lugar da $G(s)$ que aparece no integrando da equação (5), conseguimos identificar duas contribuições para a solução da equação diferencial, cada qual referente a uma das parcelas de $X(s)$. A primeira contribuição corresponde à solução da equação homogênea, dada por

$$
\begin{gathered}
x_{h}(t)=\frac{1}{2 \pi i} \int_{\gamma-i \infty}^{\gamma+i \infty} X_{h}(s) e^{s t} d s \Rightarrow \\
x_{h}(t)=\frac{1}{2 \pi i} \int_{\gamma-i \infty}^{\gamma+i \infty} \frac{x_{0} s+\left(v_{0}+\beta x_{0}\right)}{s^{2}+\beta s+\omega_{0}^{2}} e^{s t} d s
\end{gathered}
$$

onde a analiticidade do integrando nos permite deformar o caminho de integração e aplicar o teorema de Cauchy. Na Figura 1 representamos o caminho de integração, juntamente com os pólos simples do integrando, $s_{ \pm}=-\beta / 2 \pm i \omega$, sendo $\omega=\sqrt{\omega_{0}^{2}-\beta^{2} / 4}$, para o caso de amortecimento subcrítico, ou seja, quando $\omega_{0}>\beta / 2$. Aplicando o teorema de Cauchy [10], temos

$$
x_{h}(t)=2 \pi i \sum_{r e s} X_{h}(s) e^{s t}=2 \pi i\left(\frac{\tilde{f}\left(s_{+}\right)}{\tilde{g}^{\prime}\left(s_{+}\right)}+\frac{\tilde{f}\left(s_{-}\right)}{\tilde{g}^{\prime}\left(s_{-}\right)}\right)
$$

com $\sum_{\text {res }} X_{h}(s) e^{s t}$ representando a soma dos resíduos de $X_{h}(s) e^{s t}, \tilde{f}(s)=x_{0} s e^{s t}+\left(v_{0}+\beta x_{0}\right) e^{s t}$ e $\tilde{g}(s)=$ $s^{2}+\beta s+\omega_{0}^{2}$. Obtemos, assim, a conhecida solução de um oscilador harmônico amortecido

$$
\begin{gathered}
x_{h}(t)=2 R e\left(\frac{x_{0}(-\beta / 2+i \omega)+\beta x_{0}+v_{0}}{2 i \omega} e^{-\beta t / 2} e^{i \omega t}\right)= \\
A e^{-\beta t / 2} \cos (\omega t+\varphi),
\end{gathered}
$$

sendo $A=\frac{1}{2 \omega} \sqrt{4 \omega^{2} x_{0}^{2}+\left(2 v_{0}+\beta x_{0}\right)^{2}}$ e $\varphi=-\arctan \left(\frac{2 v_{0}+\beta x_{0}}{2 \omega x_{0}}\right)$, respectivamente, a amplitude inicial e a fase do movimento.

A segunda contribuição corresponde à solução particular da equação inomogênea e é proveniente do termo contendo a transformada de Laplace da força externa $F(s)$, sendo dada por

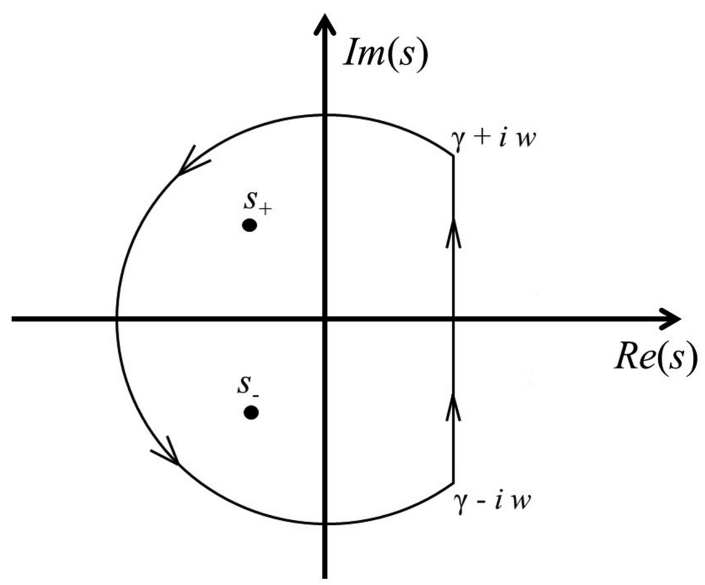

Figura 1: Caminho de integração no plano complexo da integral de Bromwich.

$$
\begin{gathered}
x_{p}(t)=\frac{1}{2 \pi i} \int_{\gamma-i \infty}^{\gamma+i \infty} X_{p}(s) e^{s t} d s= \\
\frac{1}{2 \pi i} \int_{\gamma-i \infty}^{\gamma+i \infty} \frac{F(s)}{m\left(s^{2}+\beta s+\omega_{0}^{2}\right)} e^{s t} d s .
\end{gathered}
$$

A solução final do problema dependerá da forma funcional de $F(s)$.

\section{Aplicação}

Nesta seção a metodologia de Fourier-Mellin é aplicada na solução de um problema clássico, mencionado com frequência em livros de física básica, a saber, o de uma criança periodicamente impulsionada em um balanço. É comum modelar esse problema considerando uma força senoidal aplicada pelo agente externo que empurra a criança. Todavia, uma força dessa natureza estaria aplicada de forma permanente, durante todo o tempo de oscilação, e não apenas nos curtos intervalos de tempo em que o balanço está em contato com a mão que o empurra, como ocorre na prática. Além disso, tal modelagem leva a inversões no sentido da força, algo que também não verificamos na prática, quando apenas uma pessoa, parada, empurra o balanço. Dessa forma, para modelar o sistema de modo mais realista, consideramos a força externa descrita por um trem de impulsos, todos no mesmo sentido, com mesma intesidade $J_{0}$ e atuando em intervalos de tempo muito curtos, comparados com o tempo $\tau$ que corresponde ao intervalo entre dois empurrões consecutivos. Essa força pode ser representada pela distribuição

$$
F(t)=J_{0} \sum_{n=1}^{N} \delta(t-n \tau)
$$

conhecida na literatura como pente de Dirac 11 13, pois as sucessões de impulsos no tempo fazem lembrar 
os dentes de um pente, como representada na Figura (2). A aproximação considerada $(t \rightarrow 0$ em cada um dos $n$ pulsos) será cada vez melhor quanto menor for o tempo de contato do agente externo comparado ao período de oscilação do balanço.

Aplicando a transformada de Laplace na equação (10),

$$
F(s)=J_{0} \int_{0}^{\infty} \sum_{n=1}^{N} \delta(t-n \tau) e^{-s t} d t=J_{0} \sum_{n=1}^{N} e^{-s n \tau}
$$

e substituindo na equação (9) a expressão obtida, chegamos na integral

$$
x_{p}(t)=\frac{J_{0}}{m} \frac{1}{2 \pi i} \sum_{n=1}^{N} \int_{\gamma-i \infty}^{\gamma+i \infty} \frac{e^{(t-n \tau) s}}{m\left(s^{2}+\beta s+\omega_{0}^{2}\right)} d s, t>n \tau,
$$

cujo integrando possui os mesmos polos associados ao termo transiente, que, assim como o caminho de integração, podem ser representados pelo diagrama da Figura 1. Assim, aplicando o teorema de Cauchy através da equação (7), obtemos a solução particular

$$
x_{p}(t)=\frac{J_{0}}{m \omega} \sum_{n=1}^{N} \Theta(t-n \tau) e^{-\beta(t-n \tau) / 2} \sin (\omega(t-n \tau)),
$$

que, somada à solução da equação homogênea, dada pela equação (8), fornece a solução final do problema proposto.

Observemos que a força externa possui um caráter permanente, de modo que escolhemos, $N=t_{\text {total }} / \tau$, sendo $t_{\text {total }}$ o tempo total de observação do movimento.

As equações (8) e (13) mostram que tanto a solução homogênea quanto a solução particular possuem um amortecimento exponencial $e^{-\beta t / 2}$ em sua amplitude, de modo que, para tempos suficientemente grandes, a dinâmica do sistema passa a ser fortemente influenciada por efeitos dissipativos, o que leva a uma grande perda de energia mecânica. Nas subseções a seguir veremos que

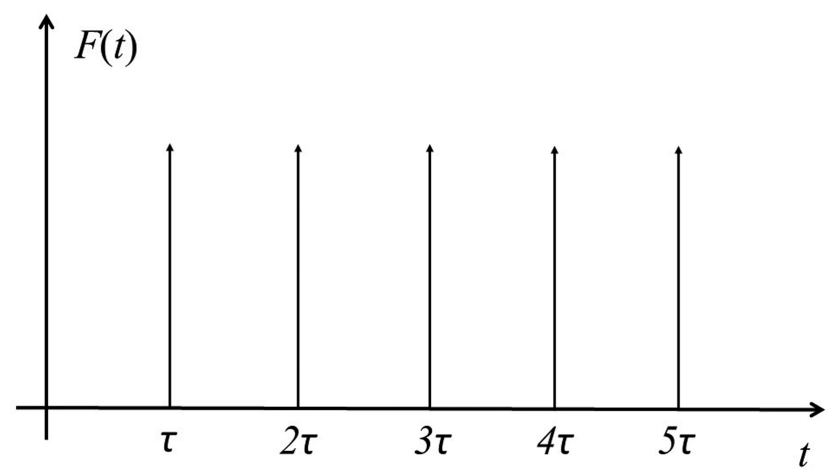

Figura 2: Representação gráfica do pente de Dirac. essa perda de energia pode ser controlada em situações nas quais o período de oscilação da força externa se aproxima do período natural de oscilação do sistema, quando os efeitos de batimento e ressonância se manifestam. Para obtenção dos resultados preparamos o sistema com os seguintes parâmetros: $x_{0}=0.1 \mathrm{~m}, v_{0}=0, \beta=0.02 \mathrm{~s}^{-1}$, $\omega_{0}=1 \mathrm{rad} / \mathrm{s}, J_{0}=0.01 \mathrm{~N} . \mathrm{s}, \tau=6 \mathrm{~s}$ e $m=0.5 \mathrm{~kg}$.

\subsection{Batimentos}

Para tempos suficientemente grandes, $t>>1 / \beta$, o movimento oscilatório passa a ser governado pela solução particular, dada pela equação 13 , de modo que podemos desprezar o termo transiente. Para melhor compreender a física do problema escrevemos essa solução como

$$
x(t) \approx \frac{J_{0}}{m \omega} \tilde{A}(t) \sin (\omega t)+\frac{J_{0}}{m \omega} \tilde{B}(t) \cos (\omega t),
$$

(onde utilizamos a propriedade $\sin (a-b)=\sin (a) \cos (b)-$ $\sin (b) \cos (a))$, tal que

$$
\tilde{A}(t)=\sum_{n=1}^{N} \Theta(t-n \tau) e^{-\beta(t-n \tau) / 2} \cos (\omega n \tau)
$$

$\mathrm{e}$

$$
\tilde{B}(t)=-\sum_{n=1}^{N} \Theta(t-n \tau) e^{-\beta(t-n \tau) / 2} \sin (\omega n \tau) .
$$

No regime considerado podemos interpretar a solução 14 como uma superposição de dois batimentos defasados de $\pi / 2$, que ocorre quando o período $\tau$ de oscilação da força externa difere ligeiramente de um múltiplo inteiro do período natural de oscilação do sistema, dado por $T=2 \pi n / \omega$. Nessa situação, as envoltórias representadas pelas equações 15 e 16 expressam o acoplamento entre a força externa, que tende a manter o batimento, e o atrito viscoso, que tende a dissipá-lo.

As Figuras 3ta e 3 b mostram o efeito do amortecimento no regime de batimento. Na primeira vemos que o amortecimento dissipa as oscilações das envoltórias, de modo que, para longos períodos de oscilação, essas envoltórias variam lentamente, $|d \tilde{A}(t) / d t|<<1 \mathrm{e}$ $|d \tilde{B}(t) / d t|<<1$, e a amplitude de oscilação se estabiliza. Nesse regime a dinâmica do sistema passa a ser governada pela superposição de duas oscilações com a mesma frequência natural $\omega$.

O domínio da solução particular para tempos suficientemente grandes está de acordo com as equações (14), (15) e (16), e reflete o fato de a solução da equação homogênea ir a zero para esses valores de tempo, como exposto no pequeno quadro da Figura 3 a, quando finalmente a solução particular é predominante. Um aspecto interessante é que, devido ao fato dos períodos de batimento ocorrerem em torno de múltiplos inteiros do 

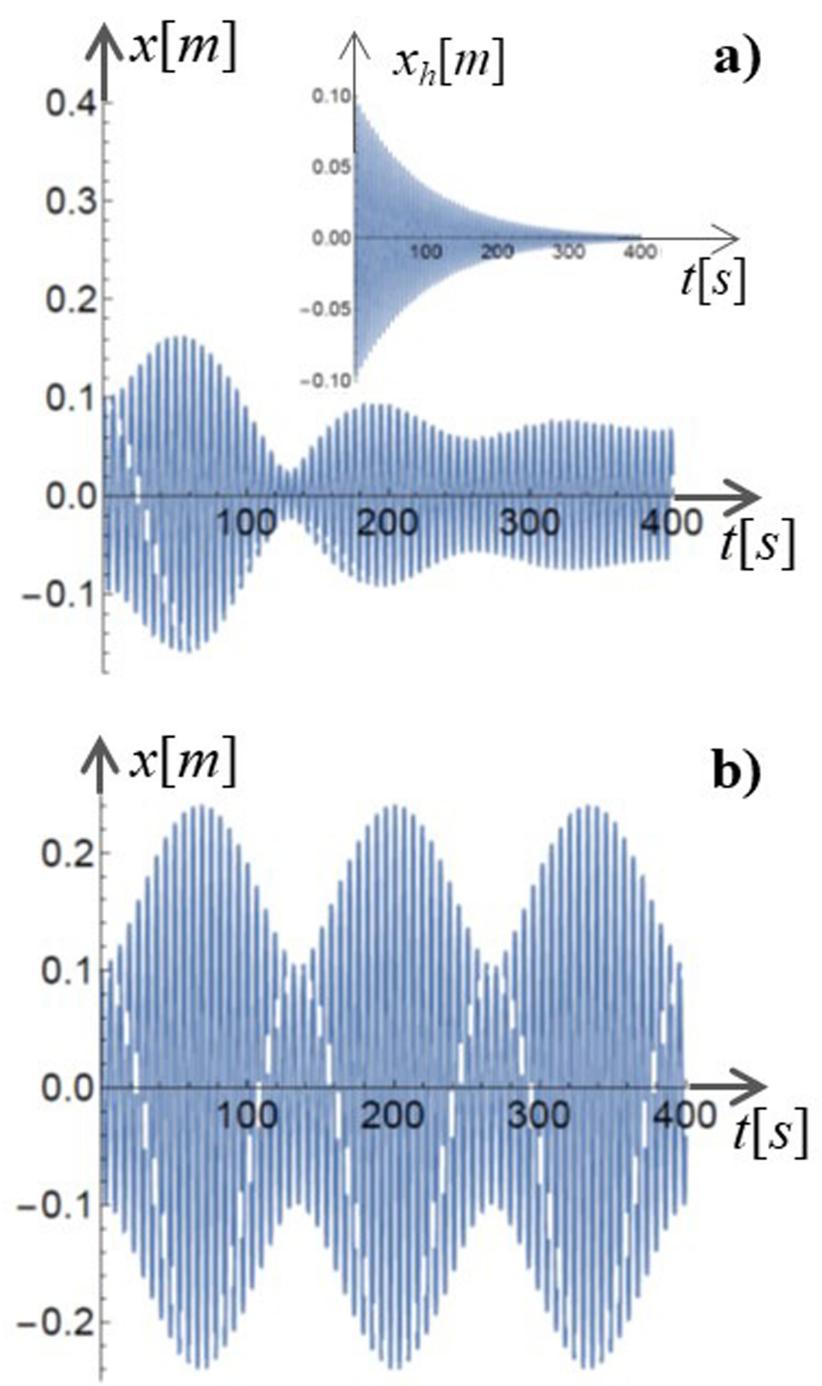

Figura 3: Posição em função do tempo. a) Consideramos a situação em que o período da força externa difere ligeiramente do período natural de oscilação $\delta \tau=|\tau-2 \pi / \omega| \approx 0.28 \mathrm{~s}$, com os efeitos de batimento e amortecimento competindo entre si. No gráfico em anexo temos a evolução do termo transiente, associado à solução da equação homogênea. b) Consideramos o amortecimento nulo $\beta=0$ e repetimos os outros parâmetros de entrada.

período natural de oscilação, podemos concluir que tal sistema possui infinitos períodos, e consequentemente infinitas frequências, em torno das quais o fenômeno do batimento se manifesta. Por outro lado, quanto maior for esse múltiplo inteiro, menor será a amplitude estabilizada, uma vez que aumentará o período de atuação dos efeitos dissipativos sem a atuação da força impulsora.

$\mathrm{Na}$ Figura 3b consideramos o amortecimento nulo, regime sob o qual o sistema evolui em termos de uma oscilação livre que se soma aos batimentos. Nesse caso as envoltórias se reduzem a $\tilde{A}(t) \rightarrow \sum_{n=1}^{N} \Theta(t-n \tau) \cos (\omega n \tau)$ e $\tilde{B}(t) \rightarrow-\sum_{n=1}^{N} \Theta(t-n \tau) \sin (\omega n \tau)$, com a solução final sendo dada por
$x(t)=A \cos (\omega t+\varphi)+\frac{J_{0}}{m \omega} \tilde{A}(t) \sin (\omega t)+\frac{J_{0}}{m \omega} \tilde{B}(t) \cos (\omega t)$, (17)

onde a solução da equação homogênea dada pela equação 8. $\operatorname{com} \beta=0$, é adicionada à solução $x(t)$ da equação (14).

\subsection{Ressonância}

No regime de ressonância o período de oscilação da força externa se iguala a um múltiplo inteiro do período natural de oscilação do sistema, $\tau \rightarrow 2 \pi n / \omega$, tal que, de acordo com as equações 15 e $16, \tilde{A}(t) \rightarrow$ $\sum_{n=1}^{N} \Theta(t-n \tau) e^{-\beta(t-n \tau) / 2}$ e $\tilde{B}(t) \rightarrow 0$. Portanto, para tempos muito longos, a posição do oscilador é dada por

$$
x(t) \approx \frac{J_{0}}{m \omega} \sum_{n=1}^{N} \Theta(t-n \tau) e^{-\beta(t-n \tau) / 2} \sin (\omega t) .
$$

Vemos a partir da equação (18) que as oscilações senoidais da solução são moduladas por uma envoltória que cresce indefinidamente na ausência de amortecimento, quando $\beta=0$, proporcionando um aumento gradual da amplitude de oscilação, que diverge para tempos grandes $(\tilde{A}(t) \rightarrow \infty$ quando $t \rightarrow \infty)$, o que caracteriza o fenômeno de ressonância mostrado na Figura 4 b. O efeito do amortecimento, introduzido a partir do parâmetro $\beta \neq 0$, por sua vez, estabiliza o aumento da envoltória a partir de um determinado tempo, como mostra o quadro principal da Figura 4 a, de tal modo que a amplitude passa a variar lentamente nesse regime, $|d \tilde{A}(t) / d t|<<1$.

A potência média transferida pela força externa ao longo de um período de oscilação $\tau$ é dada por

$$
<P(t)>=\frac{1}{\tau} \int_{t}^{t+\tau} F\left(t^{\prime}\right) v\left(t^{\prime}\right) d t^{\prime}
$$

Para tempos grandes a dinâmica do sistema é governada pela solução particular, de modo que a velocidade é obtida pela derivada da equação (13),

$$
\begin{aligned}
& v(t) \approx \frac{d x_{p}(t)}{d t} \approx \frac{J_{0}}{m} \sum_{n=1}^{N} \Theta(t-n \tau) e^{-\beta(t-n \tau) / 2} \\
& \times \cos (\omega(t-n \tau))
\end{aligned}
$$

onde usamos o fato da variação da envoltória ser muito pequena, após longos períodos de oscilação, sendo toda a variação de $x_{p}(t)$ atribuída ao fator $\sin (\omega(t-n \tau))$. Substituindo as equações (10) e 20) em (19), obtemos uma expressão para a potência média transferida pela força externa em função de seu período $\tau$ de oscilação, dada por

$$
\begin{aligned}
& <P(t)>=\frac{J_{0}^{2}}{m \tau} \sum_{n^{\prime}=1}^{N} \sum_{n=1}^{N} \Theta\left(\left(n^{\prime}-n\right) \tau\right) e^{-\beta\left(n^{\prime}-n\right) \tau / 2} \\
& \times \cos \left(\omega\left(n^{\prime}-n\right) \tau\right) .
\end{aligned}
$$



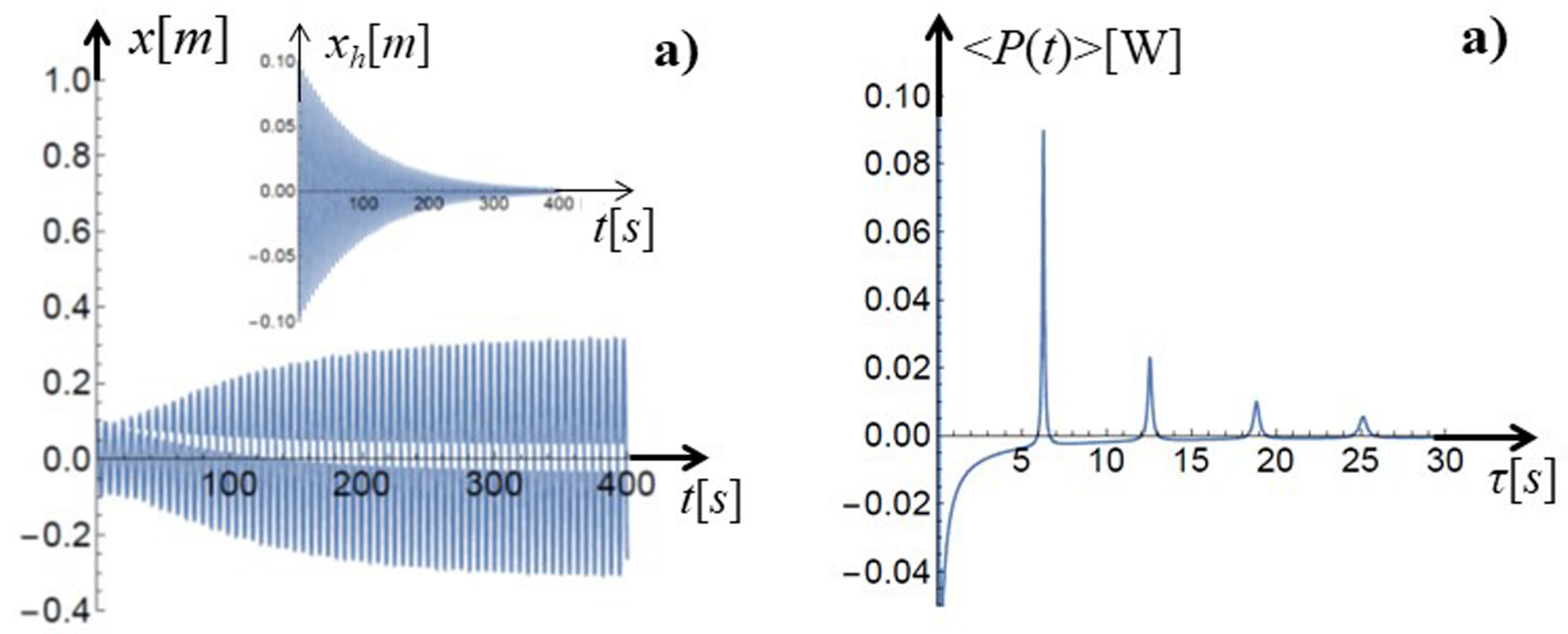

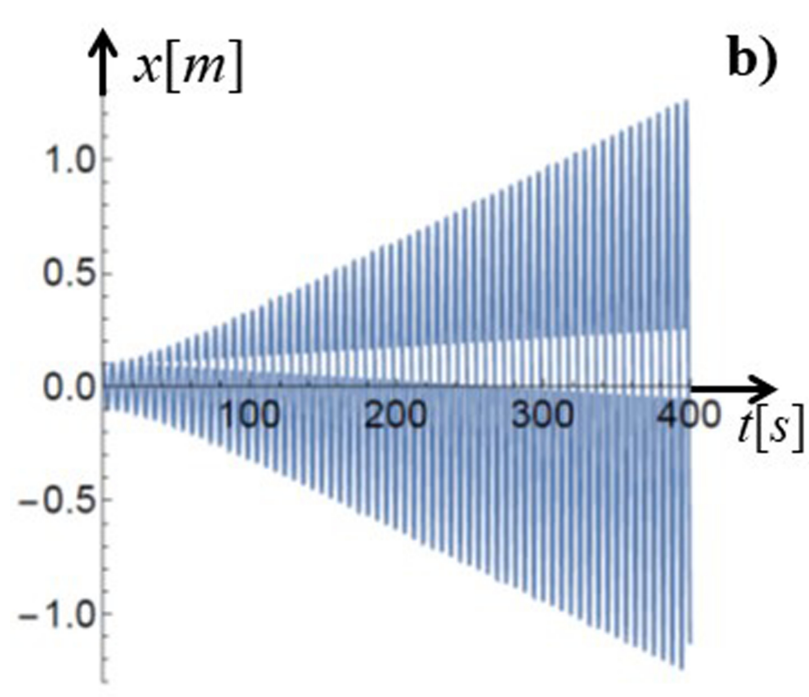

Figura 4: Posição em função do tempo. a) Consideramos a situação em que o período da força externa se iguala ao período natural de oscilação $\tau=2 \pi / \omega \approx 6.2 s$, com os efeitos de ressonância e amortecimento competindo entre si. No gráfico em anexo temos a evolução do termo transiente, associado à solução da equação homogênea. b) Consideramos o amortecimento nulo $\beta=0$ e repetimos os outros parâmetros de entrada.

A Figura 5 mostra o espectro de ressonância que se manifesta na potência média transferida pela força externa em função de seu período de oscilação. A série de picos periódicos obtida, nos quais a potência transferida é máxima, evidencia os sucessivos acessos do sistema ao regime de ressonância, que ocorrem nas inúmeras vezes em que o período da força externa se iguala a múltiplos inteiros do período natural de oscilação do sistema. A Figura 5 ta mostra que na presença de amortecimento os valores máximos atingidos pela potência média transferida diminuem de forma gradativa com aumento do número de vezes em que o regime de ressonância é acessado pelo sistema. Por outro lado, a Figura 5 b mostra

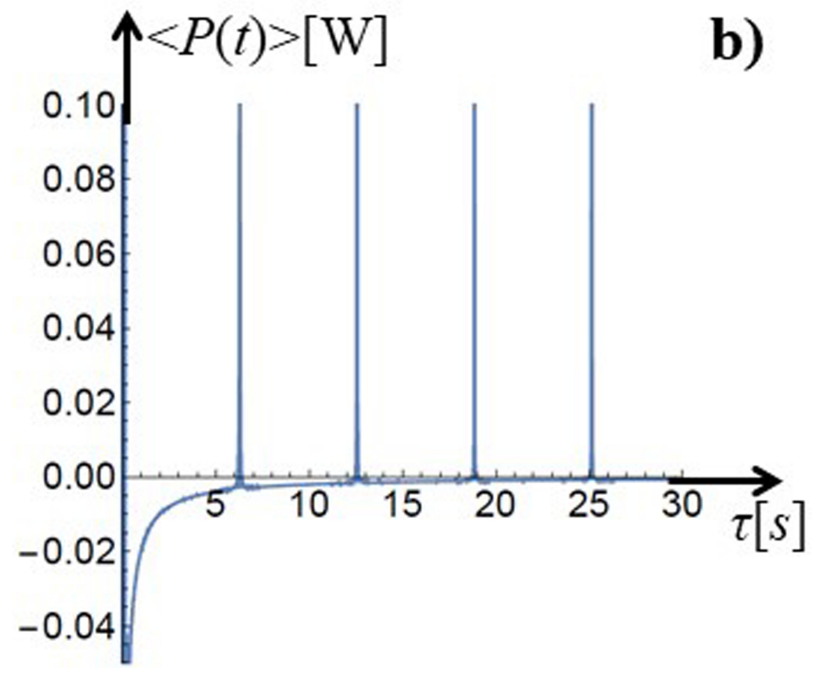

Figura 5: Espectro de potência: potência média transferida pela força externa em função do período $\tau$ de oscilação dessa força. a) Na presença de amortecimento, $\beta=0.02 \mathrm{~s}^{-1}$. b) Na ausência de amortecimento, $\beta=0$.

que na ausência de amortecimento os valores ressonantes da potência média transferida não apresentam o acentuado efeito de redução observado na Figura 5 a.

\section{Considerações Finais}

Neste trabalho apresentamos a transformada de FourierMellin como uma importante ferramenta para a solução de equações diferenciais associadas ao problema do oscilador forçado pela ação de uma força externa $F(t)$ dependente do tempo. Apresentamos uma metodologia para a aplicação dessa transformada considerando o problema geral do oscilador forçado, com a dependência temporal da força externa ainda não determinada, e com a possibilidade de amortecimento proveniente de efeitos dissipativos com dependência linear em relação à velo- 
cidade. Em seguida, aplicamos essa metodologia para solucionar o problema específico de oscilações forçadas no qual a força externa é representada por um pente de Dirac. Observamos a importância do problema proposto pelo fato de a equação diferencial a ele associada poder ser utilizada na modelagem de sistemas oscilatórios sob o efeito de impulsos externos periódicos, exemplos que vão desde a situação mais cotidiana em que uma criança é impulsionada em um balanço a possíveis aplicações em circuitos elétricos, quando submetidos a picos periódicos de tensão.

Em relação aos resultados, observamos que, de modo geral, a dinâmica do sistema é fortemente influenciada por efeitos dissipativos provenientes do amortecimento exponencial $e^{-\beta t / 2}$, presente tanto na solução particular como na solução da equação homogênea, o que acaba promovendo uma abrupta queda na amplitude de oscilação. Então, ajustamos o período de oscilação da força externa e levamos o oscilador a dois regimes de interesse, a saber, os regimes de batimento e de ressonância, ambos caracterizados por uma forte redução dos efeitos dissipativos e pela consequente estabilização da amplitude.

O regime de batimento é acessado pelo ajuste do período de oscilação da força externa a valores no entorno de múltiplos inteiros do período natural de oscilação do sistema. A solução obtida nesse regime mostra a presença de duas envoltórias responsáveis por modular as amplitudes de oscilação. Essas envoltórias incorporam tanto os efeitos de amortecimento, que tendem a diminuir a amplitude, como os efeitos provenientes da força externa, que tendem a manter os batimentos. Para tempos pequenos observamos a predominância dos efeitos dissipativos, presentes tanto na solução homogênea quanto na particular. Já para tempos suficientemente grandes, a força externa passa a desempenhar um papel importante na dinâmica do sistema, competindo com a força de atrito e promovendo a estabilização da amplitude. Verificamos uma competição análoga no regime de ressonância, que pode ser acessado ao selecionarmos o período da força externa para ser um múltiplo inteiro do período natural de oscilação do sistema. Nesse regime a amplitude também é estabilizada na presença de efeitos dissipativos, em decorrência da competição entre o termo de amortecimento e a força externa aplicada.

Por fim, mencionamos que o problema do oscilador forçado por um pente de Dirac expõe um cenário no qual o regime de ressonância se manifesta através de uma série de picos que refletem os múltiplos termos das frequências de Fourier presentes no sinal associado à força externa, cada qual contribuindo com um dos picos observados na potência média transferida.

\section{Referências}

[1] H.M. Nussenzveig, Física Básica (Editora Edgard Blucher, São Paulo, 1996), $3^{\text {a }}$ ed, v. 2.
[2] A.C. Silva e J.A. Helayël Neto, Rev. Bras. Ens. Fis. 38, 3310 (2016).

[3] A.C. Bertuola, M.S. Hussein e M.P. Pato, Rev. Bras. Ens. Fis. 27, 327 (2005).

[4] M.R. Spiegel, Transformadas de Laplace, São Paulo: Coleção Schaum (McGraw - Hill, Nova Iorque, 1979).

[5] V. Namias, American Journal of Physics 45, 624 (1977).

[6] F. Erman, M. Gadella e H. Uncu, European Journal of Physics 39, e035403 (2018).

[7] K.R. Symon, Mecânica (Editora Campus, São Paulo, 1982), $5^{\mathrm{a}}$ ed.

[8] W.E. Boyce e R.C. Diprima Equações Diferenciais Elementares e Problemas de Valores de Contorno (Editora LTC, Rio de Janeiro, 2015), 10 a ed.

[9] R.N. Bracewell e R. Bracewell, The Fourier transform and its applications (McGraw-Hill, Nova Iorque, 1986).

[10] E. Butkov, Física Matemática (Editora Ltc, Rio de Janeiro, 1988)

[11] R.S. Strichartz, A guide to distribution theory and Fourier transforms (World Scientific Publishing Company, Singapura, 2003).

[12] D. Kiang, American Journal of Physics 42, 785 (1974).

[13] O. Aouadi, Y. Chargui e M.S. Fayache, Journal of Mathematical Physics 5, e023522 (2016). 